\title{
Innovative Geospatial and Cartographic Approaches to Identification, Analysis, and Visualisation of Land Degradation
}

\author{
Řezník T. ${ }^{\mathrm{a},}$ *, Konečný M. ${ }^{\mathrm{a}}$, Charvát K. ${ }^{\mathrm{b}}$ \\ ${ }^{a}$ Masaryk University, tomas.reznik@sci.muni.cz, konecny@geogr.muni.cz \\ ${ }^{b}$ Lesprojekt-služby, charvat@lesprojekt.cz. \\ * Corresponding author
}

Keywords: Land Degradation, Visual Analytics Tools, Precision Farming, Linked Data, Environmental Policy

\begin{abstract}
:
Land degradation has increasingly been recognised as a serious environmental problem throughout the whole world. Six major threats may be identified that place soil fertility at risk, namely, soil erosion, loss of organic matter, soil biodiversity, soil compaction, soil salinity, and soil pollution. Several of those risks could be effectively identified, analysed, visualised and consequently limited by approaches originating from the geospatial domain.

The China - European Union research and innovation project "SIEUSOIL" (SIno-EU Soil Observatory for Intelligent Land Use Management), funded between 2019 and 2022, as well as the European Big Data flagship research and innovation project "DataBio" (Data-Driven Bioeconomy), funded between 2017 and 2019, aim at the above mentioned challenges of land degradation from innovative geospatial perspectives.
\end{abstract}

Such innovative approaches include local geological, geomorphological, climate, yield maps as well as global soil maps provided commonly by the European Commission (Directorate General Joint Research Centre) and the Chinese Academy of Sciences. Among the data inputs there are farm machinery tracking and interpreted satellite images. The second level of activities consists of selected standards and specification of ontologies for the integration and sharing of soil related data, facilitating their reuse and understanding. The resulting ontology and other selected models provide the basis for the integration, publication, and querying of the source datasets using Linked data as a federated layer. Besides offering an integrated view of the datasets, this layer also enables new insights through the discovery of links.

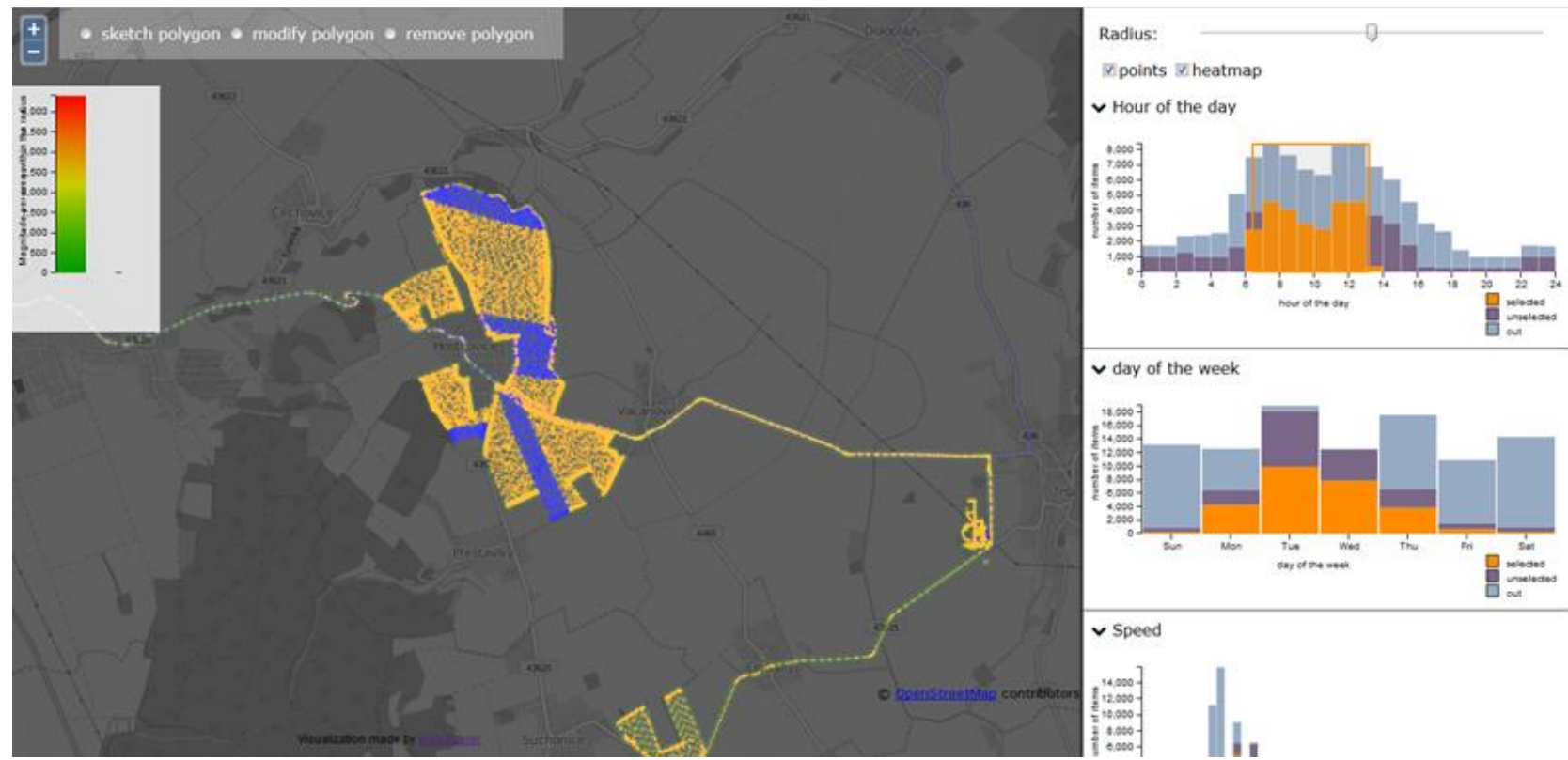

Figure 1. Interactive cartographic visualization of agricultural machinery monitoring. It allows a user to filter according to days, hours of a day, speed of a tractor, fuel consumption, etc.

From the cartographic perspective, several visualization techniques are deployed; from static maps to interactive visualisations. The main focus of visualization techniques is therefore given to the interactivity through utilizing the concept of Multiple Coordinated Views (also known as visual analytics tools) and dynamic queries to emphasize the impact of changes of various phenomena in space and time. For instance, see Figure 1 on monitoring of machinery fleet movement and especially its spatiotemporal changes that can bring new insights into the consequences of human decisions 
from many areas. Economic reasons are related to economic evidence for a farmer, including fuel consumption, efficiency of trajectory, etc. to revenue authority or subsidies management. On the other hand, ecologic motivations aim to decrease of environmental burden caused e.g. by high $\mathrm{CO}_{2}$ emissions due to a lack of movement optimisation, water pollution by nitrogen due to excessive fertilisation, etc.

The outcomes of the above mentioned research are intended as a feedback to (inter)national policies within the environmental domain including the Montreal Protocol (on substances that deplete the Ozone Layer), European Common Agricultural Policy, Nitrates Directive, Air Quality Framework Directive, Water Framework Directive, the Chinese Water Pollution Prevention Law, etc.

This research has received funding from the project of the European Union's Horizon 2020 research and innovation programme called "SIno-EU Soil Observatory for Intelligent Land Use Management" (SIEUSOIL; grant agreement not reallocated so far) and under grant agreement No 732064 called "Data-Driven Bioeconomy” (DataBio). 